ON THE DIOPHANTINE EQUATION $\sum_{i=1}^{n} x_{i} / d_{i} \equiv 0(\bmod 1)$

\author{
SUN QI AND DAQING WAN
}

(Communicated by William Adams)

Abstract. Let $d_{1}, \ldots, d_{n}$ be $n$ positive integers. The purpose of this note is to study the number of solutions and the least solutions of the following diophantine equation:

$$
\frac{x_{1}}{d_{1}}+\cdots+\frac{x_{n}}{d_{n}} \equiv 0 \quad(\bmod 1), \quad 1 \leq x_{i} \leq d_{i}-1,
$$

which arises from diagonal hypersurfaces over a finite field. In particular, we determine all the $d_{i}$ 's for which (1) has a unique solution.

Let $F_{q}$ be a finite field of $q$ elements, $c_{i}(i=1, \ldots, n)$ be nonzero elements of $F_{q}$. Suppose that $d_{i}$ divides $q-1$ for all $i$. Let $N$ be the number of solutions of the equation:

$$
c_{1} x_{1}^{d_{1}}+\cdots+c_{n} x_{n}^{d_{n}}=0, \quad x_{i} \in F_{q} .
$$

It is well known (see [1]) that

$$
\left|N-q^{n-1}\right| \leq I\left(d_{1}, \ldots, d_{n}\right)(q-1) q^{(n-2) / 2},
$$

where $I\left(d_{1}, \ldots, d_{n}\right)$ is the number of solutions of equation (1). Recently, it has been proven in [3] that

$$
\operatorname{ord}_{q}\left(N-q^{n-1}\right) \geq L\left(d_{1}, \ldots, d_{n}\right)-1,
$$

where $L\left(d_{1}, \ldots, d_{n}\right)$ is the least positive integer represented by $\sum_{i=1}^{n} x_{i} / d_{i}$ $\left(1 \leq x_{i} \leq d_{i}-1\right)$ and ord $q$ is the additive $q$-adic valuation normalized such that $\operatorname{ord}_{q} q=1$.

Thus, the archimedean estimate of $N$ is reduced to give a good upper bound on the total number $I\left(d_{1}, \ldots, d_{n}\right)$ of solutions of equation (1); while the $q$ adic estimate of $N$ is reduced to give a good lower bound for the least solution of equation (1), i.e., $L\left(d_{1}, \ldots, d_{n}\right)$. In a previous article [2], we answered the question when equation (1) is unsolvable. In the present paper, we study $I$ and $L$. In particular, we are able to characterize all the $d_{i}$ 's for which equation (1) has a unique solution. We note that there is a combinatorial formula for

Received by the editors January 2, 1990 and, in revised form, March 12, 1990.

1980 Mathematics Subject Classification (1985 Revision). Primary 11D41; Secondary 11D61, $11 \mathrm{D} 85$. 
$I\left(d_{1}, \ldots, d_{n}\right)$ (see [2]). Unfortunately, this formula does not tell much about $I$ and $L$.

Our first result is the following reduction theorem, on which our other results are based.

\section{Theorem 1.}

(i) For each $i$, define $u_{i}=\operatorname{gcd}\left(d_{i}, d_{1} \cdots d_{n} / d_{i}\right)$. Then we have the following two equalities:

$$
\begin{aligned}
& I\left(d_{1}, \ldots, d_{n}\right)=I\left(u_{1}, \ldots, u_{n}\right), \\
& L\left(d_{1}, \ldots, d_{n}\right)=L\left(u_{1}, \ldots, u_{n}\right) .
\end{aligned}
$$

(ii) For each $i$, define $v_{i}=\operatorname{gcd}\left(u_{i}, u_{1} \cdots u_{n} / u_{i}\right)$. Then we have $v_{i}=u_{i}$ for all $i$.

Part (i) of the theorem says that there is a reduction process for $I$ and $L$. Part (ii) of the theorem says that this process terminates at the second step.

Proof. Consider the equation:

$$
\frac{y_{1}}{u_{1}}+\cdots+\frac{y_{n}}{u_{n}} \equiv 0 \quad(\bmod 1), \quad 1 \leq y_{i} \leq u_{i}-1 .
$$

We claim that $x_{i}=y_{i} d_{i} / u_{i}$ gives a one-one correspondence between the solutions of equation (1) and the solutions of equation (7). Part (i) of the theorem then follows from this correspondence. To prove the claim, it is sufficient to prove that any solution $\left(x_{1}, \ldots, x_{n}\right)$ of equation (1) satisfies $x_{i}=y_{i} d_{i} / u_{i}$ for suitable integers $y_{i}(1 \leq i \leq n)$.

Let $b_{1}, \ldots, b_{n}$ be a solution of (1). Thus, there is a positive integer $z$ such that

$$
\frac{b_{1}}{d_{1}}+\cdots+\frac{b_{n}}{d_{n}}=z .
$$

Multiply both sides of (8) by $d_{1} \cdots d_{n} / u_{1}$, we have

$$
\frac{b_{1}}{u_{1}} d_{2} \cdots d_{n}+\sum_{i=2}^{n} b_{i} \frac{d_{1}}{u_{1}} \frac{d_{2} \cdots d_{n}}{d_{i}}=z \frac{d_{1}}{u_{1}} d_{2} \cdots d_{n} .
$$

Since $\left(d_{1} / u_{1}, d_{2} \cdots d_{n} / u_{1}\right)=1$, from (9) we have

$$
b_{1} \equiv 0 \quad\left(\bmod \frac{d_{1}}{u_{1}}\right) \text {. }
$$

Similarly, we have

$$
b_{i} \equiv 0 \quad\left(\bmod \frac{d_{i}}{u_{i}}\right) .
$$

Thus, $b_{i}=d_{i} y_{i} / u_{i}$ for some integers $y_{i}(1 \leq i \leq n)$, and the claim is proved.

To prove the second part of the theorem, we need to verify

$$
u_{i}=\operatorname{gcd}\left(u_{i}, u_{1} \cdots u_{n} / u_{i}\right) \quad(1 \leq i \leq n) .
$$


For any given prime number $l$, let $h_{i}=\operatorname{ord}_{l}\left(d_{i}\right)$ (for simplicity of notation, we suppress the dependence of $h_{i}$ on $l$ ). Then, we have

$$
\begin{gathered}
\operatorname{ord}_{l} u_{i}=\min \left(h_{i}, \sum_{j \neq i} h_{j}\right), \\
\operatorname{ord}_{l} u_{1} \cdots u_{n} / u_{i}=\sum_{j \neq i} \min \left(h_{j}, \sum_{k \neq j} h_{k}\right) .
\end{gathered}
$$

Thus, (10) holds if and only if the following inequality holds for all prime numbers $l$ and all $i \quad(1 \leq i \leq n)$,

$$
\min \left(h_{i}, \sum_{j \neq i} h_{j}\right) \leq \sum_{j \neq i} \min \left(h_{j}, \sum_{k \neq j} h_{k}\right) .
$$

Case I. $h_{i} \leq h_{j}$ for some $j \neq i$. In this case, we have

$$
\min \left(h_{i}, \sum_{j \neq i} h_{j}\right)=h_{i} \leq \min \left(h_{j}, \sum_{k \neq j} h_{k}\right) .
$$

Clearly, the right term of (12) is less than or equal to the right term of (11).

Case II. $h_{i} \geq \max _{j} h_{j}$ for all $j \neq i$. In this case,

$$
\min \left(h_{i}, \sum_{j \neq i} h_{j}\right) \leq \sum_{j \neq i} h_{j} \leq \sum_{j \neq i} \min \left(h_{j}, \sum_{k \neq j} h_{k}\right) .
$$

Thus, (11) holds in this case, too. The theorem is proved.

As a corollary of Theorem 1 , we have the following estimates for $I$ and $L$.

Theorem 2. For all $j(1 \leq j \leq n)$, the following two inequalities hold:

$$
\begin{gathered}
I\left(d_{1}, \ldots, d_{n}\right) \leq \prod_{i \neq j}\left(u_{i}-1\right), \\
L\left(d_{1}, \ldots, d_{n}\right) \geq \frac{1}{u_{1}}+\cdots+\frac{1}{u_{n}} .
\end{gathered}
$$

Part (ii) of Theorem 1 shows that if one repeats the process of (5) and (6), one does not get a better bound.

Proof. Consider equation (7). (15) is trivial by Theorem 1. To prove (14), it suffices to show that for each choice of $y_{i}(i \neq j)$ there is at most one $y_{j}$ satisfying equation (7). We may suppose that $j=1$. If for a given $y_{i}$ $\left(1 \leq y_{i} \leq u_{i}-1, i=2,3, \ldots, n\right)$ there are two choices for $y_{1}$, say $y_{1}$ and $z_{1}$, satisfying (7), then one has

$$
\left(y_{1}-z_{1}\right) / u_{1} \equiv 0 \quad(\bmod 1) .
$$

This implies that $y_{1}=z_{1}$. Thus, (14) is true.

The next result describes all $d_{i}$ 's for which equation (1) has no solutions. 
Theorem 3. Let $u_{i}=\operatorname{gcd}\left(d_{i}, d_{1} \cdots d_{n} / d_{i}\right) \quad(1 \leq i \leq n)$. The following conditions are equivalent.

(a) $I\left(d_{1}, \ldots, d_{n}\right)=0$.

(b) $L\left(d_{1}, \ldots, d_{n}\right)=+\infty$.

(c) Either some $u_{i}=1$, or let $u_{i_{j}}(j=1, \ldots, k)$ be all the even integers among the $u_{i}$ 's; then $k$ is odd and $u_{i_{j}}=2$ for all $j$ except $u_{i_{l}}=2^{t}$ $(t>0)$ for one $l$.

In [2], we gave a necessary and sufficient condition for $I=0$ in terms of the $d_{i}$ 's. Unfortunately, that condition is not very simple. In contrast, the new condition (in terms of the $u_{i}^{\prime}$ 's) given in Theorem 3 is much simpler.

Proof. The equivalence of (a) and (b) is trivial. We now prove that (c) $\Rightarrow(a)$. If the first condition of (c) holds, i.e., some $u_{i}=1$, then (7) has no solutions. Theorem 1 shows that $I\left(d_{1}, \ldots, d_{n}\right)=0$. If the second condition of (c) holds, then for any solution $y_{i}(i=1, \ldots, n)$ of (7), we must have $y_{i_{j}}=1$ and $k$ even. This contradicts the assumption that $k$ is odd. Thus, $I\left(d_{1}, \ldots, d_{n}\right)=0$. Next, we prove $(\mathrm{a}) \Rightarrow(\mathrm{c})$. Assuming $I\left(d_{1}, \ldots, d_{n}\right)=0$, the result in [2] shows that one of the following conditions holds:

(i) For some $i, \operatorname{gcd}\left(d_{i}, d_{1} \cdots d_{n} / d_{i}\right)=1$.

(ii) Let $d_{i_{j}}(j=1, \ldots, k)$ be all the even integers among the $d_{i}$ 's; then $k$ is odd, $d_{i_{1}} / 2, \ldots, d_{i_{k}} / 2$ are pairwise prime, and any $d_{i_{j}}$ is prime to any odd numbers among the $d_{i}$ 's.

If (i) is true, then $u_{i}=1$ and the first condition of (c) holds. We now suppose that (ii) is true. If $k=1$, one checks that $u_{i_{1}}=1$. Hence, the first condition of (c) holds. If $k>1$, it is easy to see that the second condition of (c) is satisfied. This proves that (a) implies (c). Theorem 3 is proved.

The last result describes all $d_{i}$ 's for which equation (1) has a unique solution. Theorem 4. Let $n>1$. Let $u_{i}=\operatorname{gcd}\left(d_{i}, d_{1} \cdots d_{n} / d_{i}\right)(1 \leq i \leq n)$. The following conditions are equivalent.

(a) $I\left(d_{1}, \ldots, d_{n}\right)=1$.

(b) $n$ is even and $u_{i}=2$ for all $i$ except $u_{j}=2^{k} \quad(k>0)$ for one $j$.

Proof. First, (b) $\Rightarrow$ (a). Without loss of generality, we suppose that $u_{1}=\cdots=$ $u_{n-1}=2$ and $u_{n}=2^{k}$ for some $k>0$. If $y_{i}=b_{i}(1 \leq i \leq n)$ give a solution of (7), then one must have $b_{1}=\cdots=b_{n-1}=1$ and $b_{n}=2^{k-1}$. Thus (7) has a unique solution.

Next, we prove $(\mathrm{a}) \Rightarrow(\mathrm{b})$. Let $y_{1}, \ldots, y_{n}$ be the unique solution of equation (7). It is clear that $u_{i}-y_{i}(1 \leq i \leq n)$ also satisfy (7). By uniqueness of solution, we have $y_{i}=u_{i} / 2$ for all $i$. We claim that $\operatorname{gcd}\left(u_{i}, u_{j}\right)=2$ for all $i \neq j$. To prove the claim, we let $d=\operatorname{gcd}\left(u_{i}, u_{j}\right)>2$ for some $i \neq j$. Then 
the equation

$$
\frac{z_{i}}{u_{i}}+\frac{z_{j}}{u_{j}} \equiv 0 \quad(\bmod 1), \quad 1 \leq z_{l} \leq u_{l}-1,
$$

has exactly $d-1>1$ solutions. Furthermore, it is easy to see that one can choose a solution $z_{l}$ such that $z_{l} \neq u_{l} / 2 \quad(l=i, j)$. Then $y_{i}+z_{i}\left(\bmod u_{i}\right)$, $y_{j}+z_{j}\left(\bmod u_{j}\right), y_{l}(l \neq i, j)$ give rise to a new solution of $(7)$ contradicting with the assumption on uniqueness.

By part (ii) of Theorem 1, we have

$$
\operatorname{gcd}\left(u_{i}, u_{1} \cdots u_{n} / u_{i}\right)=u_{i} \quad(1 \leq i \leq n) .
$$

The above claim and (18) show that all the $u_{i}$ 's are powers of 2 . One more application of the claim $\operatorname{gcd}\left(u_{i}, u_{j}\right)=2$ implies that $u_{i}=2$ for all $i$ except $u_{j}=2^{k} \quad(k>0)$ for one $j$. Theorem 4 is proved.

From the first part of the proof of Theorem 4, we have

Corollary 5. Assume the $d_{i}$ 's satisfy one of the equivalent conditions in Theorem 4 ; then

$$
L\left(d_{1}, \ldots, d_{n}\right)=n / 2
$$

\section{ACKNOWLEDGMENT}

This paper was written while the first author was visiting the University of Washington. He would like to thank Neal Koblitz and Ralph Greenberg for their generous support.

\section{REFERENCES}

1. K. Ireland and M. Rosen, A classical introduction to modern number theory, Graduate Texts in Math., vol. 84, Springer-Verlag, New York, 1982.

2. Sun Qi and Daqing Wan, On the solvability of the equation $\sum_{i=1}^{n} x_{i} / d_{i} \equiv 0(\bmod 1)$ and its application, Proc. Amer. Math. Soc. 100 (1987), 220-224.

3. Daqing Wan, Zeros of diagonal equations over finite fields, Proc. Amer. Math. Soc. 103 (1988), 1049-1052. China

Department of Mathematics, Sichuan University, Chengdu, People's Republic of

Department of Mathematics, The University of Washington, Seattle, Washington 98195 